\title{
Interação entre salinidade da água de irrigação e adubação nitrogenada na cultura da berinjela
}

\author{
Francisco de A. de Oliveira ${ }^{1}$, José F. de Medeiros $^{2}$, Rita de C. Alves ${ }^{3}$, \\ Paulo S. F. Linhares ${ }^{4}$, Arthur M. A. de Medeiros ${ }^{5} \&$ Mychelle K. T. de Oliveira $^{6}$
}

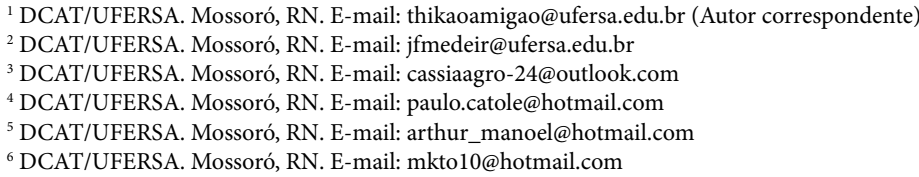

Palavras-chave:

Solanum melongena L.

estresse salino

nitrogênio

\begin{abstract}
R E S U M O
Propôs-se, neste trabalho, avaliar a interação de níveis de salinidade da água de irrigação associada a doses de nitrogênio sobre a produção de frutos de berinjela. O experimento foi conduzido em ambiente protegido do Departamento de Ciências Ambientais e Tecnológicas - UFERSA. O experimento obedeceu a um delineamento de blocos casualizados em esquema fatorial 4 x 6, com quatro repetições e quatro níveis de condutividade elétrica da água de irrigação $\left(0,5 ; 2,4\right.$ e $\left.6 \mathrm{dS} \mathrm{m} \mathrm{m}^{-1}\right)$ além de seis doses de nitrogênio $\left(5,10,15,20,25\right.$ e $\left.30 \mathrm{~g} \mathrm{planta}^{-1}\right)$. Seis colheitas foram realizadas nas quais se analisaram as seguintes variáveis: número de frutos, comprimento, diâmetro e massa média de frutos e produção total de frutos por planta. Após a análise estatística dos dados verificou-se efeito negativo da salinidade sobre todas as variáveis analisadas. Doses de nitrogênio acima de $5 \mathrm{~g} \mathrm{planta}^{-1}$ não aumentaram a produção de frutos da berinjela mas aumentaram o efeito deletério da salinidade sobre o seu rendimento. A eficiência agronômica do nitrogênio é reduzida com o aumento na dose de $\mathrm{N}$ e da salinidade.
\end{abstract}

Key words:

Solanum melongena L. saline stress

nitrogen

\section{Interaction between water salinity and nitrogen fertilization on the eggplant crop}

\begin{abstract}
A B S T R A C T
The objective of this study was to evaluate the interaction of salinity of irrigation water levels associated with nitrogen doses on yield of eggplant crop. The experiment was conducted in a greenhouse of the Department of Environmental Sciences and Technology - UFERSA. The experiment followed a randomized block design in a $4 \times 6$ factorial, with four replications, with four levels of electrical conductivity of irrigation water $\left(0.5,2,4\right.$ and $\left.6 \mathrm{dS} \mathrm{m}^{-1}\right)$ and six levels of nitrogen $\left(5,10,15,20,25\right.$ and $\left.30 \mathrm{~g} \mathrm{plant}^{-1}\right)$. Six measurements were conducted, in which the following variables were analysed: number of fruits, length, diameter and mean fruit weight, and total yield per plant. After statistical analysis of the data, it was verified that there was a negative effect of salinity on all variables. Nitrogen levels above $5 \mathrm{~g} \mathrm{plant}^{-1}$ did not increase fruit yield of eggplant, and increased the deleterious effect of salinity on the yield of eggplant. The agronomic efficiency of nitrogen is reduced with increasing dose of $\mathrm{N}$ and salinity.
\end{abstract}

\section{INTRODUÇÃO}

A cultura da berinjela (Solanum melongena L.) tem-se expandido consideravelmente nos últimos anos visto que seus frutos apresentam características peculiares no uso em dietas fitoterápicas, com substâncias medicinais relevantes (Raigón et al., 2008); em razão dessas propriedades o consumo de berinjela vem aumentando entre os brasileiros.

Um dos principais fatores que afetam a produção da berinjela é a qualidade da água utilizada na irrigação visto que o estresse salino provoca alterações morfofisiológicas, tais como: desequilíbrio nutricional, redução na condutância estomática e menores proporções nas taxas de transpiração, fotossíntese e concentração interna de $\mathrm{CO}_{2}$ nas folhas, resultando morfologicamente em diminuição de biomassa em planta e no rendimento de frutos (Moura et al., 2004; Bosco et al., 2009; Wu et al., 2012; Silva et al., 2013).

A berinjela é uma cultura moderadamente sensível à salinidade, apresentando salinidade limiar de $1,5 \mathrm{dS} \mathrm{m}^{-1} \mathrm{e}$ perda de rendimento de $4,4 \%$ por aumento unitário da salinidade (Unlukara et al., 2010); no entanto, a sensibilidade da berinjela à salinidade pode variar de acordo com o material genético utilizado e com as condições de cultivo (Parida \& Das, 2005).

$\mathrm{O}$ nitrogênio se destaca como um dos nutrientes mais significativos para as plantas pois desempenha função estrutural e faz parte de diversos compostos orgânicos vitais para o vegetal, 
como aminoácidos, proteínas e prolina, entre outros, elevando a capacidade de ajustamento osmótico das plantas à salinidade e aumenta a resistência das culturas ao estresse hídrico e salino (Parida \& Das, 2005). Desta forma, o manejo adequado da adubação nitrogenada pode ser uma alternativa para atenuar o efeito da salinidade sobre as plantas.

São poucos estudos sobre a interação entre nitrogênio e salinidade em diferentes culturas, sendo encontrados resultados divergentes. Melo et al. (2011) verificaram, trabalhando com a cultura do meloeiro, que sob estresse salino as plantas não responderam à adubação nitrogenada; entretanto, Oliveira et al. (2010) não constataram interação significativa entre salinidade e adubação nitrogenada na cultura do girassol.

$\mathrm{Na}$ literatura brasileira existem poucos trabalhos sobre adubação nitrogenada na cultura da berinjela. Castro et al. (2005) verificaram, trabalhando com adubação nitrogenada utilizando como fonte a cama de frango, que a berinjela responde até a dose de $395 \mathrm{~kg} \mathrm{ha}^{-1}$ de nitrogênio enquanto Trani et al. (2011) recomendam a aplicação $290 \mathrm{~kg} \mathrm{ha}^{-1}$ de nitrogênio para 12.500 plantas.

Em outros países são encontrados relatos divergentes sobre a resposta da cultura ao nitrogênio, nos quais máximos rendimentos de frutos foram obtidos com doses variando de 70 a $185 \mathrm{~kg} \mathrm{ha}^{-1}$ de nitrogênio (Pal et al., 2002; Aminifard et al., 2010; Moraditochaee et al., 2011).

A divergência referida acima demonstra a necessidade do desenvolvimento de mais estudos com adubação na cultura da berinjela, especialmente em diferentes condições ambientais. Diante do exposto este trabalho foi desenvolvido com o objetivo de se avaliar o efeito da adubação nitrogenada na produção de berinjela irrigada com águas salinas.

\section{Material e Métodos}

O experimento foi realizado de outubro de 2011 a março de 2012 na área experimental do Departamento de Ciências Ambientais e Tecnológicas da Universidade Federal Rural do Semi-Árido (UFERSA) em Mossoró, RN, localizada nas coordenadas geográficas de $5^{\circ} 11^{\prime} 31^{\prime \prime}$ de latitude Sul e 370 20' $40^{\prime}$ de longitude Oeste de Greenwich e altitude média de $18 \mathrm{~m}$.

De acordo com a classificação climática de Köeppen, o clima de Mossoró é do grupo BSwh', isto é, tropical semiárido muito quente e com estação chuvosa ocorrendo no verão-outono apresentando temperatura média de $27,4{ }^{\circ} \mathrm{C}$, precipitação pluviométrica anual muito irregular, com média de $673,9 \mathrm{~mm}$ e umidade relativa do ar de 68,9\% (Carmo Filho \& Oliveira, 1995).

Utilizou-se o delineamento em blocos casualizado seguindo arranjo fatorial $4 \times 6$, com quatro repetições, resultando no total de 96 unidades experimentais. Os tratamentos foram resultantes da combinação de quatro níveis de salinidade da água de

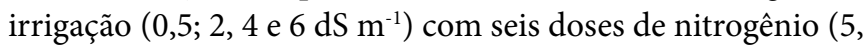
$10,15,20,25$ e 30 g planta $\left.^{-1}\right)$.

As unidades experimentais se constituíram de um vaso com capacidade para $20 \mathrm{~L}$, contendo uma planta em cada vaso. Cada unidade experimental foi preenchida com 18 litros de solo acondicionados de forma a apresentar densidade aparente próxima à observada no solo na condição natural (Tabela 1).

Utilizaram-se mudas de berinjela híbrido 'Ciça', produzidas em bandejas de poliestireno expandido com capacidade para 128 células contendo substrato de fibra de coco e húmus de minhoca, na proporção 1:1. O transplantio foi realizado utilizando-se uma muda por vaso quando apresentava de quatro a cinco folhas definitivas, o que ocorreu por volta dos 25 dias após a semeadura. Os vasos foram dispostos em quatro fileiras espaçadas $1,5 \mathrm{~m}$ com espaçamento de $0,5 \mathrm{~m}$ entre vasos, equivalente à população de 13.333 plantas por hectare.

Além da adubação nitrogenada foram aplicados $\mathrm{P}_{2} \mathrm{O}_{5}, \mathrm{~K}_{2} \mathrm{O}$, Ca e $\mathrm{Mg}$, nas doses de 6, 25, 10 e 2 g planta $^{-1}$, respectivamente, distribuídas ao longo do experimento em intervalos semanais, de acordo com a marcha de absorção da cultura (Trani et al., 2011). Para o preparo das soluções de fertirrigação se utilizaram os seguintes fertilizantes: fosfato monoamônico (MAP), fosfato monopotássico $\left(\mathrm{KH}_{2} \mathrm{PO}_{4}\right)$, cloreto de potássio $(\mathrm{KCl})$, ureia $\left(\mathrm{CO}\left(\mathrm{NH}_{2}\right)_{2}\right)$ nitrato de potássio $\left(\mathrm{KNO}_{3}\right)$, nitrato de cálcio $\left(\mathrm{Ca}\left(\mathrm{NO}_{3}\right)_{2}\right)$ e sulfato de magnésio $\left(\mathrm{MgSO}_{4}\right)$.

Aplicações foliares de micronutrientes foram realizadas em intervalos semanais utilizando-se produto comercial apresentando a mistura sólida de EDTA-chelated nutrientes contendo $0,28 \% \mathrm{Cu}, 7,5 \% \mathrm{Fe}, 3,5 \% \mathrm{Mn}, 0,7 \% \mathrm{Zn}, 0,65 \%$ B e 0,3\% Mo; durante o ciclo foram feitas adubações foliares à base de cálcio e boro, elementos imprescindíveis na fase de frutificação.

Material de solo classificado como Argissolo Vermelho Amarelo (EMBRAPA, 2006) foi utilizado e coletado na camada de $0-20 \mathrm{~cm}$ de profundidade, em área não cultivada localizada no Campus da UFERSA. O material coletado foi secado ao ar e posteriormente peneirado em malha de $2,0 \mathrm{~mm}$ e analisado quimicamente (Tabela 1).

Com vista à salinidade $S_{1}-0,5 \mathrm{dS} \mathrm{m}^{-1}$ foi utilizada água proveniente do sistema de abastecimento do campus da UFERSA cujas análises físicas e químicas determinaram as seguintes características: $\mathrm{pH}=8,30 ; \mathrm{CE}=0,50 \mathrm{dS} \mathrm{m}{ }^{-1} ; \mathrm{Ca}^{2+}=$ 3,$10 ; \mathrm{Mg}^{2+}=1,10 ; \mathrm{K}^{+}=0,30 ; \mathrm{Na}^{+}=2,30 ; \mathrm{Cl}^{-}=1,80 ; \mathrm{HCO}_{3}^{-}=3,00$ $\mathrm{e} \mathrm{CO}_{3}{ }^{2-}=0,20\left(\mathrm{mmol}_{\mathrm{c}} \mathrm{L}^{-1}\right)$.

$\mathrm{Na}$ preparação das demais salinidades foi adicionada uma mistura de sais de $\mathrm{NaCl}, \mathrm{CaCl}_{2} \cdot 2 \mathrm{H}_{2} \mathrm{O}$ e $\mathrm{MgCl}_{2} \cdot 6 \mathrm{H}_{2} \mathrm{O}$ em água coletada em poço profundo localizado no Campus central da UFERSA, mantendo-se a proporção equivalente de 7:2:1 (Medeiros, 1992).

Tabela 1. Características físicas e químicas do solo utilizado no experimento

\begin{tabular}{|c|c|c|c|c|c|c|c|c|c|c|c|c|c|c|c|c|}
\hline \multicolumn{9}{|c|}{ Características químicas } & \multicolumn{8}{|c|}{ Características físicas } \\
\hline \multirow{2}{*}{$\mathrm{pH}$} & \multirow{2}{*}{$\begin{array}{l}\text { M.0. } \\
\text { (\%) }\end{array}$} & \multirow{2}{*}{$\begin{array}{c}P \\
\left(\mathrm{mg} \mathrm{dm}^{-3}\right)\end{array}$} & $\mathrm{K}^{+}$ & $\mathrm{Na}^{+}$ & $\mathrm{Ca}^{+2}$ & $\mathrm{Mg}^{+2}$ & $\mathrm{Al}^{+3}$ & $\mathrm{H}^{+}$ & \multicolumn{3}{|c|}{ Fração granulométrica $\left(\mathrm{g} \mathrm{kg}^{-1}\right)$} & \multirow{2}{*}{$\begin{array}{l}\text { Classe } \\
\text { textural }\end{array}$} & \multicolumn{2}{|c|}{ Umidade $\left(\mathrm{g} \mathrm{g}^{-1}\right)$} & \multicolumn{2}{|c|}{ Densidade $\left(\mathrm{kg} \mathrm{dm}^{3}\right)$} \\
\hline & & & & & (cmo) & $\left.\mathrm{dm}^{-3}\right)$ & & & Areia & Silte & Argila & & CC & PMP & Ds & Dp \\
\hline 5,7 & 1,05 & 2,20 & 0,14 & 0,13 & 0,40 & 0,60 & 0,25 & 3,05 & 707,2 & 172,2 & 120,6 & $\mathrm{FA}$ & 0,15 & 0,06 & 1,53 & 2,68 \\
\hline
\end{tabular}

FA - Franco arenoso; CC - Capacidade de campo para $\psi_{\mathrm{m}}=-10 \mathrm{kPa}$; PMP - Ponto de murcha permanente para $\psi_{\mathrm{m}}=-1500 \mathrm{kPa}$; Ds - Densidade do solo; DP - Densidade de partículas 
Para determinar a concentração de sais a serem dissolvidos em determinada quantidade de água salinizada para se obter a condutividade elétrica desejada, realizou-se calibração preliminar por meio de um diagrama de dispersão plotandose os valores de concentração de fertilizantes versus os de condutividade elétrica, seguindo metodologia utilizada por Dias et al. (2007); a partir da curva obtida verificou-se a necessidade da dissolução de $630 \mathrm{~g}$ de sais para adição de $1 \mathrm{dS} \mathrm{m}^{-1}$ à água utilizada no preparo das soluções.

A irrigação foi realizada através de sistema de gotejamento utilizando-se frequência de uma vez até os 50 dias após o tranplantio e de duas vezes ao dia: uma pela manhã e outra no final da tarde, dos 50 DAT até o final do experimento (120 dias após o transplantio). Este procedimento ocorreu em virtude da baixa capacidade de retenção de água no solo (Tabela 1) e porque aos $50 \mathrm{DAT}$ eram perceptíveis sinais de murchamento nas plantas.

Adotou-se o sistema de irrigação por gotejamento utilizando-se emissores tipo microtubo cujo fornecimento de água foi realizado através de reservatório (vasos plásticos com capacidade para $80 \mathrm{~L}$ ) suspensa sobre cavaletes de forma a se obter uma carga hidráulica inicial de 1,0 m.

O sistema de distribuição de água foi composto de quatro linhas laterais de tubos flexíveis com diâmetro de $16 \mathrm{~mm}$, uma para cada fileira de vasos, sendo instalados os microtubos nas linhas laterais, espaçados $0,5 \mathrm{~m}$, correspondente a um emissor em cada vaso. Foram utilizados emissores de $0,50 \mathrm{~m}$ de comprimento definido em testes para estabelecimento do comprimento obtendo-se vazão média de $1,6 \mathrm{~L} \mathrm{~h}^{-1}$.

Para minimizar o efeito da redução de carga hidráulica sobre a vazão dos emissores as irrigações eram iniciadas sempre com os reservatórios em sua capacidade máxima de água de forma que, para atender ao suprimento hídrico das plantas, não era necessário aplicar toda a água do reservatório.

O consumo de água pelas plantas não foi contabilizado; no entanto, para garantir a reposição da água evapotranspirada as irrigações eram suspensas após ser observado início de drenagem nos vasos, não ocorrendo fração de lixiviação significativa em nenhum dos tratamentos.

Os tratos culturais consistiram na retirada dos brotos que surgiram antes da inserção da primeira flor, tutoramento para promover a condução das plantas e aplicações preventivas com fungicida e inseticidas.

Seis colheitas de frutos foram realizadas, a primeira aos 70 dias após o transplantio e as seguintes em intervalos de 10 dias. Após a contabilização dos frutos (NFR), realizaram-se as determinações de suas duas dimensões medindo o comprimento longitudinal (CMFR) utilizando-se uma régua graduada $(\mathrm{cm})$ e o diâmetro dos frutos (DMFR), através de um paquímetro digital $(\mathrm{mm})$; em seguida, os frutos foram pesados para determinação da massa fresca (MMFR) (g) e da produção de frutos (PROD) por planta (g planta $\left.{ }^{-1}\right)$ em balança de precisão.

A partir da produção de frutos e das doses de nitrogênio determinou-se a eficiência agronômica do nitrogênio (EAN), definida pela relação entre a produção de frutos e a quantidade de nitrogênio aplicada por planta (Aujla et al., 2007).
Os dados obtidos foram submetidos à análise de variância e de regressão os quais foram ajustados aos modelos polinomiais de primeiro e segundo graus. Para as interações significativas realizou-se o desdobramento da análise de variância considerando-se isoladamente os fatores níveis de salinidade e adubação nitrogenada.

\section{Resultados e Discussão}

Comprovou-se efeito significativo da interação entre os fatores níveis de salinidade e adubação nitrogenada para o número de frutos por planta (NFR), de forma que foram ajustadas equações de regressão individuais para cada nível de salinidade e de nitrogênio.

Na Figura 1 é mostrado o efeito da salinidade sobre o NFR dentro de cada dose de nitrogênio na qual se pode perceber que os dados apresentaram melhor ajuste à equação linear $\mathrm{e}$ decrescente mas, apesar de apresentar a mesma tendência, a redução no NFR em decorrência do aumento da salinidade da água de irrigação foi maior nas plantas que receberam doses 15 e $30 \mathrm{~g}$ planta $^{-1}$, as quais apresentaram reduções médias de 0,86 frutos por planta, por aumento unitário da salinidade da água de irrigação resultando em perdas totais de 52,7 e 54,4\%, respectivamente, na maior salinidade em comparação com os resultados encontrados na salinidade de $0,5 \mathrm{dS} \mathrm{m}^{-1}$.

Pode-se observar ainda, na Figura 1, que o efeito deletério da salinidade sobre o NFR foi menor nas plantas que receberam a menor dose de $\mathrm{N}$ ( $5 \mathrm{~g} \mathrm{planta}^{-1}$ ) ocorrendo perda total de aproximadamente $26,6 \%$ na maior salinidade.

É provável que o decréscimo no número de frutos por planta seja devido ao aumento da salinidade em decorrência da alteração no potencial osmótico reduzindo o consumo de água pelas plantas e, em consequência, nutrientes diminuindo, assim, o índice de pegamento dos frutos. Resultados semelhantes obtiveram Chartzoulakis \& Loupassaki (1997) e Marques et al. (2012) para a cultura da berinjela, além de outros autores para outras hortaliças, como tomate (Medeiros et al., 2012), pimentão (Leonardo et al., 2008) e meloeiro (Melo et al., 2011).

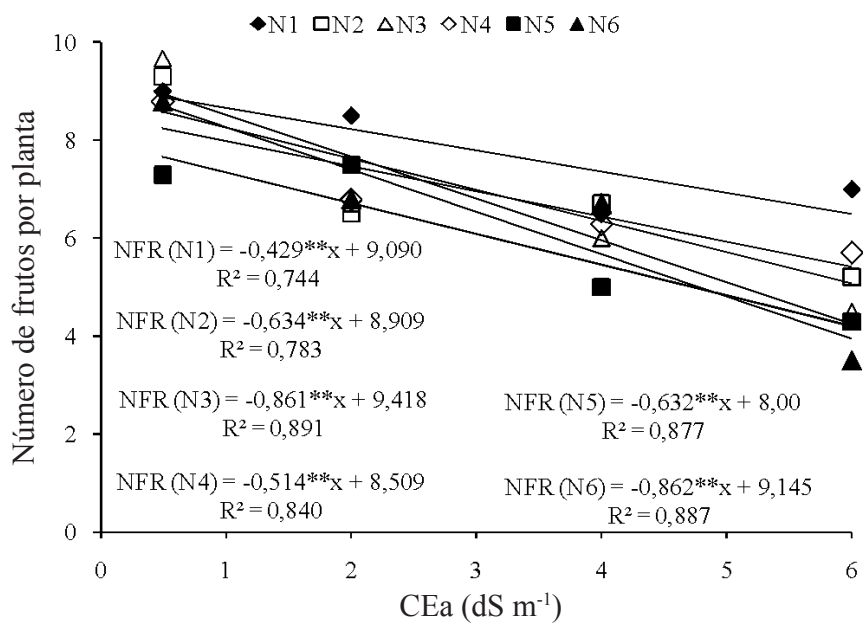

Figura 1. Número de frutos de berinjela em função da salinidade da água de irrigação em plantas submetidas a diferentes doses de nitrogênio 
Com referência ao efeito as doses de N sobre o NFR verificou-se, em cada nível de salinidade, que houve resposta apenas nas plantas irrigadas com água de maior salinidade (6 $\mathrm{dS} \mathrm{m}^{-1}$ ), sendo observada resposta linear e negativa de forma que menor NFR ocorreu na maior dose de N (30 g planta $^{-1}$ ), com aproximadamente 2,8 frutos por planta correspondente à redução total de 49,6\% em comparação com o NFR observado na dose de 5 g planta $^{-1}$ de nitrogênio (Figura 2).

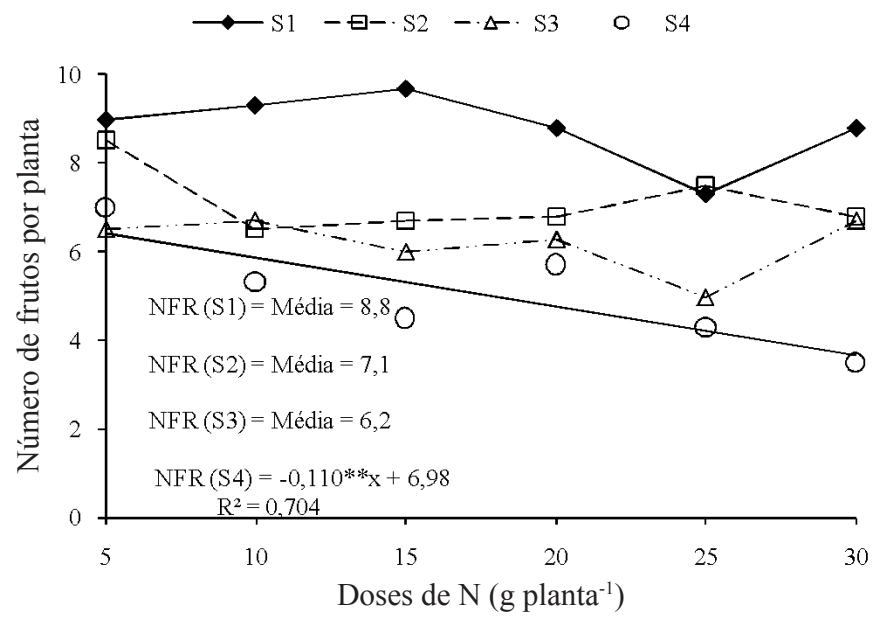

Figura 2. Número de frutos de berinjela em função da adubação nitrogenada em plantas submetidas a diferentes níveis de salinidade da água de irrigação

Para as demais salinidade não houve efeito da adubação nitrogenada sobre o NFR obtendo-se valores médios de $8,8(0,5$ $\left.\mathrm{dS} \mathrm{m}^{-1}\right), 7,1\left(2 \mathrm{dS} \mathrm{m}^{-1}\right)$ e $6,2\left(4 \mathrm{dS} \mathrm{m}^{-1}\right)$ frutos por planta (Figura 2). O efeito negativo da adubação nitrogenada sobre o NFR quando se utilizou água mais salina ocorreu, provavelmente, pelo maior acúmulo de sais no solo, provocado pela aplicação de água salina aliada ao excesso fertilizante.

De acordo com Sat \& Saimbhi (2003) doses elevadas de nitrogênio podem retardar significativamente o florescimento de berinjela e aumentar o número de dias necessários para a fixação dos frutos podendo, assim, reduzir o número de frutos em um período de avaliação, conforme observado no presente trabalho.

Para o comprimento médio de frutos (CMFR) verificouse efeito isolado dos fatores, de forma que foram ajustadas apenas uma equação de regressão à análise do efeito da salinidade (Figura 3A) e uma equação para o efeito da adubação nitrogenada (Figura 3B). Verificou-se resposta linear e negativa ao aumento da salinidade desde que os frutos de maior comprimento foram obtidos na menor salinidade $\left(0,5 \mathrm{dS} \mathrm{m}^{-1}\right)$, apresentando comprimento médio de $12,5 \mathrm{~cm}$ enquanto na maior salinidade se obtiveram frutos com comprimento médio de $9,7 \mathrm{~cm}$ resultando em decréscimo de aproximadamente 22,1\% (Figura 3A).

Verificou-se, também, resposta linear e negativa para o comprimento dos frutos quanto à adubação nitrogenada, com maior valor ocorrendo na menor dose de $\mathrm{N}\left(5 \mathrm{~g} \mathrm{planta}^{-1}\right)$ independente da salinidade da água de irrigação, com CMFR de $11,9 \mathrm{~cm}$, e redução total de $12,7 \%$ na dose de $\mathrm{N}$ equivalente a
A.

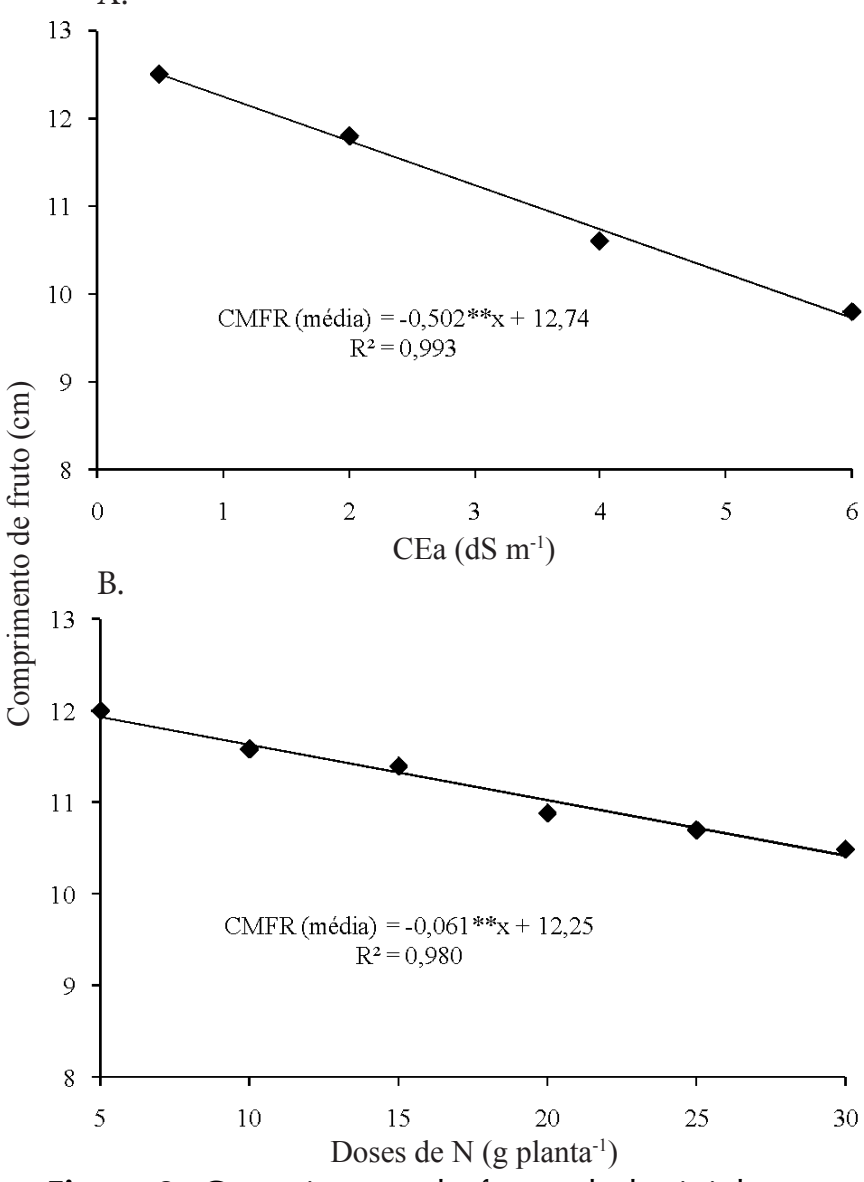

Figura 3. Comprimento de frutos de berinjela em função da salinidade da água de irrigação (A) e da adubação nitrogenada (B)

30 g planta $^{-1}$ (Figura 3B). Resultados semelhantes foram obtidos por Bozorgi (2012) avaliando o efeito da adubação nitrogenada sobre a cultura da berinjela, que também obtiveram maior número de frutos e comprimento de frutos com a aplicação de 5,25 g planta $^{-1}$.

$\mathrm{O}$ aumento da salinidade da água de irrigação afetou o diâmetro médio de frutos (DMFR) apresentando, inicialmente, efeito positivo até a salinidade de $2,48 \mathrm{dS} \mathrm{m}^{-1}(6,65 \mathrm{~cm})$ e redução a partir deste nível de maneira que, na maior salinidade ( $6 \mathrm{dS}$ $\mathrm{m}^{-1}$ ) se obtiveram menores valores com DMFR de $6,6 \mathrm{~cm}$ (Figura 4A). Não se constatou resposta significativa para a adubação nitrogenada tendo-se obtido, entre as doses de nitrogênio, frutos com DMFR médio de $6,45 \mathrm{~cm}$.

Também não houve efeito significativo da adubação nitrogenada sobre a massa fresca de frutos (MFFR) sendo encontrada MFFR média de 211,15 $\mathrm{g}_{\text {fruto }}{ }^{-1}$; no entanto, observou-se resposta significativa para a salinidade da água de irrigação ocorrendo efeito linear e negativo de vez que os frutos mais pesados foram obtidos nas plantas irrigadas com água de salinidade $0,5 \mathrm{dS} \mathrm{m}^{-1}$ e a MFFR reduziu com o aumento da salinidade tendo-se encontrado valores menores no maior nível salino ( $6 \mathrm{dS} \mathrm{m}^{-1}$ ), com MFFR média de 180,8 $\mathrm{g}$ fruto $^{-1}$, correspondendo à redução total de $25,7 \%$, entre essas salinidades (Figura 4B). 


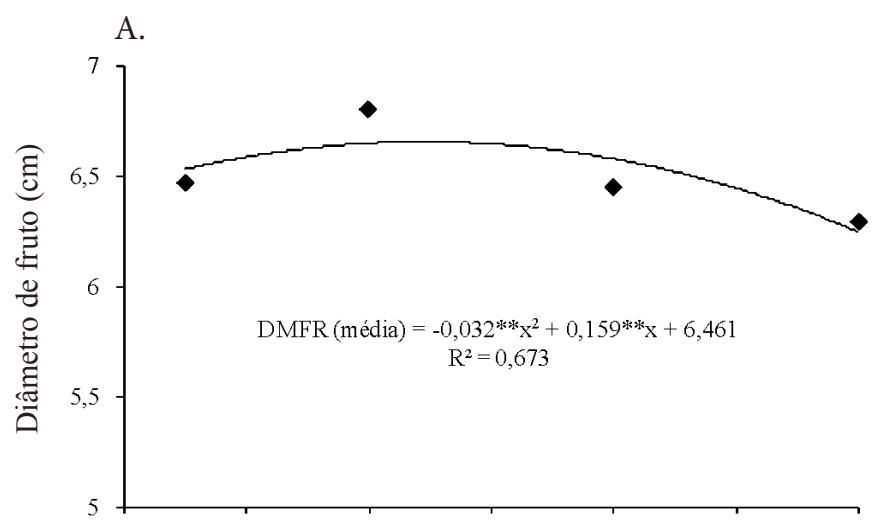

B.

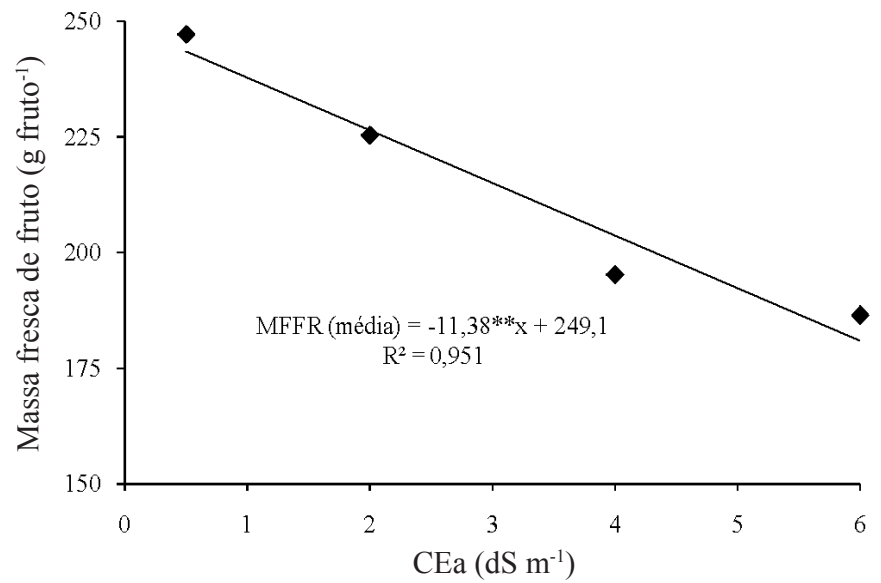

Figura 4. Diâmetro médio (A) e massa fresca de frutos de berinjela (B) em função da salinidade da água de irrigação

Silva et al. (2013) também constataram redução significativa na massa fresca de frutos de berinjela em resposta ao aumento na salinidade do solo. De modo semelhante, Leonardo et al. (2008) também observaram, trabalhando com a cultura do pimentão, relação inversa entre salinidade e massa fresca de frutos.

Quanto à produção total de frutos verificou-se resposta significativa à salinidade da água de irrigação, porém a resposta foi variável de acordo com a adubação nitrogenada. Para a maioria das doses de nitrogênio a produção de frutos reduziu significativa e linearmente com o aumento da salinidade, de forma que as maiores produções de frutos ocorreram na menor salinidade, com PROD médias de 1921,$2 ; 2007,4 ; 1650,6 ; 1614,8$ e 1561,4 g planta $^{-1}$ para as doses de $\mathrm{N}$ correspondentes a 5, 10, 15, 20, 25 e 30 g planta $^{-1}$, respectivamente (Figura 5).

Analisando o efeito da salinidade dentro de cada nível de nitrogênio, verifica-se que a PROD apresentou comportamento semelhante para os níveis de 10 e 25 g planta $^{-1}$, com resposta linear e negativa além de perdas totais variando de 46,4 a $61,0 \%$, respectivamente, correspondendo a perdas relativas de aproximadamente 8,4 a $11,2 \%$ por aumento unitário na condutividade elétrica da água de irrigação (Figura 5). Para a dose de $15 \mathrm{~g}_{\text {planta }}{ }^{-1}$ verificou-se que, apesar de ter havido redução na PROD com o aumento na salinidade houve tendência de estabilização na PROD a partir da salinidade de $4,0 \mathrm{dS} \mathrm{m}^{-1}$, de forma que, para este nível de $\mathrm{N}$, os dados foram ajustados à equação quadrática (Figura 5).

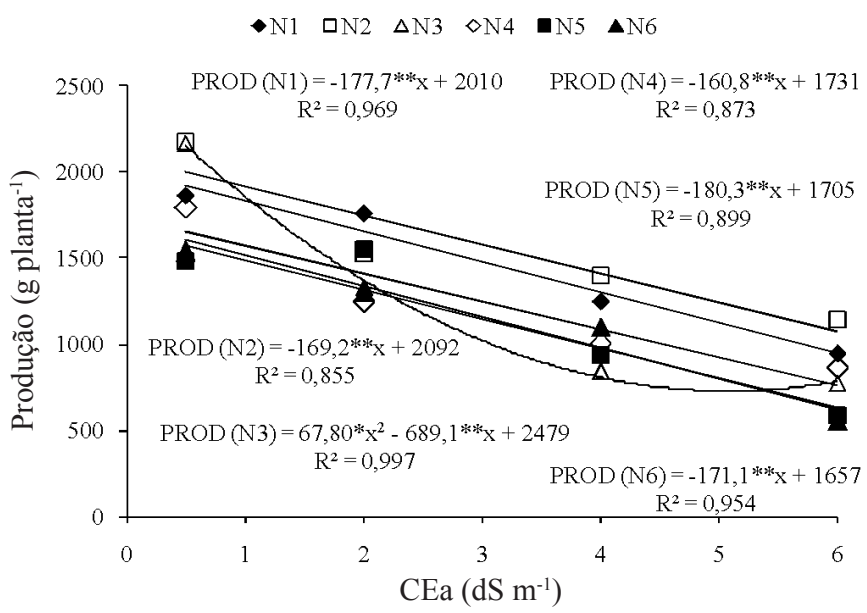

Figura 5. Produção de frutos de berinjela em função da salinidade da água de irrigação em plantas submetidas a diferentes doses de nitrogênio

De acordo com a literatura, a berinjela é classificada como moderadamente sensível à salinidade, apresentando redução no rendimento com salinidade a partir de $1,5 \mathrm{dS} \mathrm{m}^{-1}$ e perda relativa de 4,4\% para cada aumento unitário de condutividade elétrica (Unlukara et al., 2010). Trabalhando com o mesmo híbrido utilizado neste trabalho ("Ciça"), Silva et al. (2013) verificaram que a redução na produção da cultura da berinjela em estufa foi, em média, de 8,65\% por incremento de $1 \mathrm{dS} \mathrm{m}^{-1}$ na salinidade do solo acima, portanto, da salinidade limiar, que foi de 1,71 $\mathrm{dS} \mathrm{m}^{-1}$; pode-se constatar, então, que esses resultados estão bem próximos aos obtidos no presente trabalho.

Ocorreu efeito significativo das doses de nitrogênio sobre a produção de frutos; apesar disto, a resposta das plantas foi variável de acordo com a salinidade da água de irrigação. Nas plantas irrigadas com água de menor salinidade verificou-se resposta positiva ao aumento nas doses de nitrogênio até o nível de $11 \mathrm{~g} \mathrm{planta}^{-1}$, obtendo-se produção de $2.045 \mathrm{~g} \mathrm{planta}^{-1}$, decrescendo a partir desta dose de forma que os dados foram ajustados à equação quadrática. A partir desta dose verificou-se reposta negativa de maneira que a menor produção de frutos ocorreu na maior dose de $\mathrm{N}$ (30 g planta $\left.^{-1}\right)$, com PROD média de $1.394 \mathrm{~g} \mathrm{planta}^{-1}$ (Figura 6).

Melo et al. (2011) também constataram, avaliando a interação entre salinidade e adubação nitrogenada na cultura do meloeiro, resposta à adubação nitrogenada apenas nas plantas irrigadas com água de menores salinidades.

Para as plantas irrigadas com água de salinidade $2 \mathrm{dS} \mathrm{m}^{-1}$, esses autores verificaram reduções na produção de frutos com o aumento nas doses de nitrogênio obtendo os menores valores com a aplicação de $22 \mathrm{~g} \mathrm{planta}^{-1}$ de nitrogênio; obtiveram, ainda, produção de frutos de aproximadamente $1.322 \mathrm{~g} \mathrm{planta}^{-1}$ após essas doses verificou-se tendência de aumento na produção dos frutos, de forma que nessas salinidades os dados foram ajustados às equações quadráticas (Figura 6).

Para a salinidade $4,0 \mathrm{dS} \mathrm{m}^{-1}$ não foi possível obter ajuste satisfatório para os modelos utilizados neste trabalho (linear e quadrático), para a qual se obteve PROD média de $1.090 \mathrm{~g}$ planta $^{-1}$ (Figura 6). 


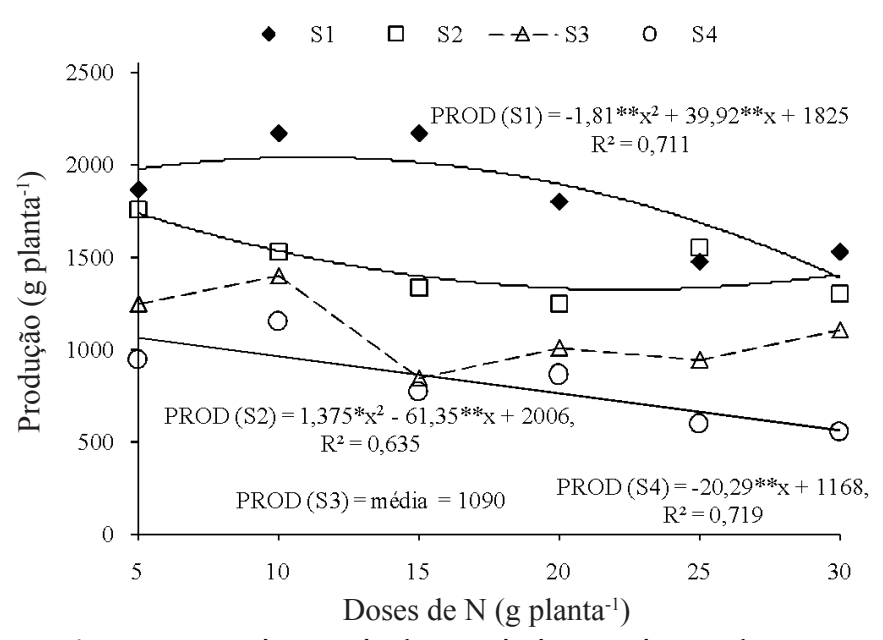

Figura 6. Produção de frutos de berinjela em função da adubação nitrogenada em plantas submetidas a diferentes níveis de salinidade da água de irrigação

Pode-se observar ainda, na Figura 6, que as plantas irrigadas com água de maior salinidade $\left(6 \mathrm{dS} \mathrm{m}^{-1}\right)$ apresentaram resposta linear e negativa ao aumento nas doses de nitrogênio, de forma que a maior produção de frutos ocorreu com a aplicação de 5 g planta $^{-1}$ de nitrogênio, com PROD média de 1.168 g planta $^{-1}$ enquanto na maior dose de nitrogênio se obteve a menor produção de frutos (média de $568 \mathrm{~g}_{\text {planta }}{ }^{-1}$ ).

Considerando o espaçamento adotado neste trabalho (1,5 x $0,5 \mathrm{~m}$ ) correspondendo à população de 13.333 plantas por hectare, verifica-se que a dose de $\mathrm{N}$ de 5 g planta $^{-1}$, equivalente a aproximadamente $67 \mathrm{~kg} \mathrm{ha}^{-1} \mathrm{de} \mathrm{N}$, está próxima dos resultados encontrados por outros autores trabalhando com a cultura da berinjela, a exemplo de Aminifard et al. (2010) e Moraditochaee et al. (2011) que obtiveram maior rendimento de berinjela com a aplicação de $70 \mathrm{~kg} \mathrm{ha}^{-1}$ de nitrogênio.

$\mathrm{Na}$ Figura 7 se encontra o efeito dos tratamentos sobre a eficiência agronômica do nitrogênio (EAN), na qual se pode perceber que houve grande variação na EAN entre os tratamentos estudados. De forma geral, a EAN foi reduzida com o aumento nas doses de $\mathrm{N}$, independente da salinidade da água de irrigação (Figura 7).

O maior valor de EAN ocorreu na dose de $5 \mathrm{~g} \mathrm{planta}^{-1}$ na salinidade de $0,5 \mathrm{dS} \mathrm{m}^{-1}$, na qual se obteve a produção de $373 \mathrm{~g}$ de fruto por $\mathrm{g}$ de $\mathrm{N}$ enquanto a menor EAN ocorreu nas plantas

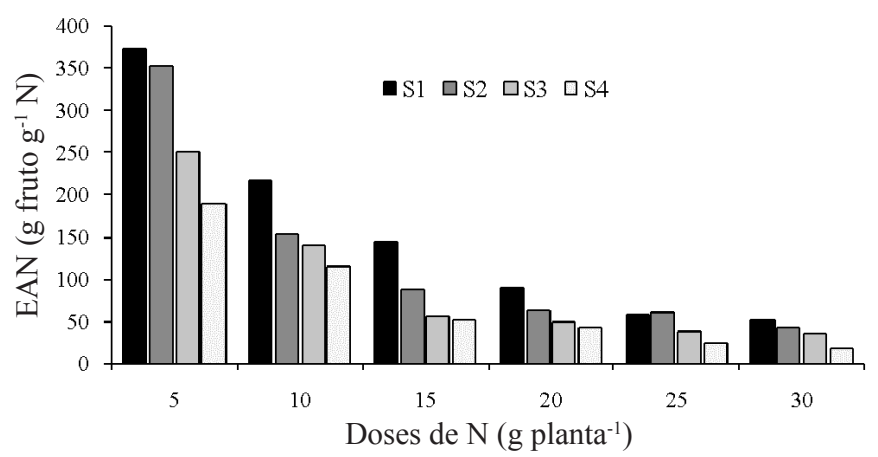

Figura 7. Eficiência agronômica do nitrogênio (EAN) da berinjela em função de doses de nitrogênio e salinidade da água de irrigação que receberam a dose de $30 \mathrm{~g} \mathrm{planta}^{-1} \mathrm{e}$ foram irrigadas com água de salinidade $6 \mathrm{dS} \mathrm{m}^{-1}$ (18,4 $\mathrm{g}$ fruto por $\mathrm{g}$ de $\left.\mathrm{N}\right)$. Observou-se, também, que a EAN foi reduzida de acordo com o aumento da salinidade independente da dose de nitrogênio (Figura 7).

Comparando os níveis extremos de salinidade $(0,5$ e 6 $\mathrm{dS} \mathrm{m}^{-1}$ ) verificou-se redução na EAN variando de 47 a $64 \%$ nas doses de 10 e 11 g planta $^{-1}$ de N. Analisando a redução na EAN de acordo com o aumento das doses de $\mathrm{N}$, foram observadas perdas de 86,$3 ; 87,7 ; 85,3$ e 90,3\%, para as salinidades 0,$5 ; 2,4$ e $6 \mathrm{dS} \mathrm{m}^{-1}$ (Figura 7).

Esses resultados divergem, em parte, dos obtidos por Aujla et al. (2007) os quais, trabalhando com métodos de irrigação e níveis de nitrogênio, constataram aumento na EAN com o aumento até a dose de $150 \mathrm{~kg} \mathrm{ha}^{-1}$ no entanto, é oportuno ressaltar que esses autores trabalharam em condições de campo nas quais as plantas apresentaram maior desenvolvimento do sistema radicular e, em contrapartida, capacidade de explorar maior volume de solo e absorver mais nutrientes.

Resultados semelhantes foram obtidos por Oliveira (2012) com a cultura do pimentão e Oliveira et al. (2008) trabalhando com a cultura do meloeiro que também verificaram que a EAN foi reduzida com o aumento na dose de nitrogênio.

Os resultados obtidos neste trabalho evidenciam a importância do manejo adequado da adubação nitrogenada na cultura da berinjela sobretudo quando o uso de água salina para irrigação é inevitável.

\section{Conclusões}

1. A interação entre os fatores estudados (salinidade da água de irrigação x níveis de nitrogênio) foi significativa para número de frutos e produção.

2. A salinidade da água de irrigação afetou negativamente o número de frutos, comprimento, diâmetro e massa média de frutos e produção total de frutos por planta.

3. Elevadas doses de nitrogênio provocam redução no rendimento de berinjela e potencializam os efeitos deletérios da salinidade da água de irrigação sobre as variáveis número de frutos, produção e eficiência agronômica da adubação nitrogenada.

4. Quando se utiliza água com nível de salinidade de até 4 $\mathrm{dS} \mathrm{m}^{-1}$ recomenda-se a dose de $10 \mathrm{~g}$ de $\mathrm{N}$ por planta.

5. Elevadas doses de nitrogênio e o uso de água salina reduzem a eficiência agronômica do nitrogênio na cultura da berinjela.

6. A partir da dose de $5 \mathrm{~g}$ de $\mathrm{N}$ por planta a aplicação de $\mathrm{N}$ não proporciona incremento na Eficiência da Adubação Nitrogenada, independentemente da salinidade da água de irrigação.

\section{Agradecimentos}

Os autores agradecem Instituto Nacional de Ciência e Tecnologia em Salinidade (INCTSal) pelo apoio financeiro necessário para o desenvolvimento desta pesquisa. Ao Grupo de Pesquisas em Irrigação e Nutrição de Plantas (IRRIGANUTRI) pela disponibilidade da infraestrutura utilizada. 


\section{Literatura Citada}

Aminifard, M. H.; Aroiee, H.; Fatemi, H.; Ameri, A.; Karimpour, S. Responses of eggplant (Solanum melongena $\mathrm{L}$.) to different rates of nitrogen under field conditions. Journal of Central European Agriculture, v.11, p.453-458, 2010.

Aujla, M. S.; Thind, H. S.; Buttar, G. S. Fruit yield and water use efficiency of eggplant (Solanumme longema L.) as influenced by different quantities of nitrogen and water applied through drip and furrow irrigation. Scientia Horticulturae, v.112, p.142-148, 2007.

Bosco, M. R. O.; Oliveira, A. B.; Hernandez, F. F. F.; Lacerda, C. F. de. Influência do estresse salino na composição mineral da berinjela. Revista Ciência Agronômica, v.40, p.157-164, 2009.

Bozorgi, H. R. Study effects of nitrogen fertilizer management under nano iron chelate foliar spraying on yield and yield components of eggplant (Solanum melongena L.). Journal of Agricultural and Biological Science, v.7, p.233-237, 2012.

Carmo Filho, F.; Oliveira, O. F. Mossoró: Um município do semi-árido nordestino, caracterização climática e aspecto florístico. Mossoró: ESAM, 1995. 62p. Coleção Mossoroense, série $\mathrm{B}$.

Castro, M. C.; Almeida, D. L.; Ribeiro, R. L. D.; Carvalho, J. F. Plantio direto, adubação verde e suplementação com esterco de aves na produção orgânica de berinjela. Pesquisa Agropecuária Brasileira, v.40, p.495-502, 2005.

Chartzoulakis, K. S.; Loupassaki, M. H. Effects of $\mathrm{NaCl}$ salinity on germination, growth, gas exchange and yield of greenhouse eggplant. Agricultural Water Management, v.32, p.215-225, 1997.

Dias, N. da S.; Duarte, S. N.; Teles Filho, J. F.; Yoshinaga, R. T. Salinização do solo por aplicação de fertilizantes em ambiente protegido. Irriga, v.12, p.135-143, 2007.

EMBRAPA - Empresa Brasileira de Pesquisa Agropecuária. Sistema brasileiro de classificação de solos. 2.ed. Rio de Janeiro: EMBRAPA, 2006. 306p.

Leonardo, M.; Broetto, F.; Villas-Bôas, R. L.; Marchese, J. A.; Tonin, F. B.; Regina, M. Estado nutricional e componentes da produção de plantas de pimentão conduzidas em sistema de fertirrigação durante indução de estresse salino em cultivo protegido. Bragantia, v.67, p.883-889, 2008.

Marques, D. J.; Broetto, F.; Lobato, A. K. S.; Silva, E. C.; Carvalho, J. G.; Ávila, F. W.; Alves, G. A. R.; Andrade, I. P. Photosynthetic pigments, nitrogen status, and flower behavior in eggplant exposed to different sources and levels of potassium. Scientific Research and Essays, v.8, p.67-74, 2012.

Medeiros, J. F. de. Qualidade da água de irrigação utilizada nas propriedades assistidas pelo "GAT" nos Estados do RN, $\mathrm{PB}, \mathrm{CE}$ e avaliação da salinidade dos solos. Universidade Federal da Paraíba, Campina Grande: UFPB, 1992. 173p. Dissertação Mestrado

Medeiros, P. R. F.; Duarte, S. N.; Uyeda, C. A.; Silva, E. F. F.; Medeiros, J. F. de. Tolerância da cultura do tomate à salinidade do solo em ambiente protegido. Revista Brasileira de Engenharia Agrícola e Ambiental, v.16, p.5155, 2012.
Melo, T. K.; Medeiros, J. F. de; Espínola Sobrinho, E.; Figueirêdo, V. B.; Pereira, V. C.; Campos, M. S. Evapotranspiração e produção do melão Gália irrigado com água de diferentes salinidades e adubação nitrogenada. Revista Brasileira de Engenharia Agrícola e Ambiental, v.15, p.1235-1242, 2011.

Moraditochaee, M.; Bozorgi, H. R.; Halajisani. Effects of vermicompost application and nitrogen fertilizer rates on fruit yield and several attributes of eggplant (Solanum melongena L.) in Iran. World Applied Sciences Journal, v.15, p.174-178, 2011.

Moura, D. C. M.; Carvalho, J. A.; Gomes, L. A. A. Evapotranspiração da cultura da berinjela irrigada com diferentes concentrações de sais na água. Engenharia Agrícola, v.15, p.1-6, 2004.

Oliveira, F. A. Cultivo do pimentão em ambiente protegido utilizando diferentes manejos de fertirrigação. Piracicaba: ESALQ/USP, 2012. 222p. Tese Doutorado

Oliveira, F. A.; Medeiros, J. F. de; Lima, C. J. G. S.; Dutra, I.; Oliveira, M. K, T. de. Eficiência agronômica da fertirrigação nitrogenada e potássica na cultura do meloeiro nas condições do semi-árido nordestino. Caatinga, v.21, p.5-11, 2008.

Oliveira, F. A.; Oliveira, F. R. A.; Campos, M. S.; Oliveira, M. K. T.; Medeiros, J. F. de; Silva, O. M. P. Interação entre salinidade e fontes de nitrogênio no desenvolvimento inicial da cultura do girassol. Revista Brasileira de Ciências Agrárias, v.5, p.479-484, 2010.

Pal, S.; Saimbhi, M. S.; Bal, S.S. Effect of nitrogen and phosphorus levels on growth and yield of brinjal hybrid (Solanum melongena L.). Journal of Vegetation Science. v.29, p. 90-91, 2002.

Parida, A. K.; Das, A. B. Salt tolerance and salinity effects on plants: A review. Ecotoxicology and Environmental Safety, v.60, p.324-349, 2005.

Raigón, M. D.; Prohens, J.; Munõz-Falcón, J. E.; Nuez, F. Comparison of eggplant landraces and commercial varieties for fruit content of phenolics, minerals, dry matter and protein. Journal of Food Composition and Analysis, v.21, p.370-376, 2008.

Sat, P.; Saimbhi, M S. Effect of varying levels of nitrogen and phosphorus on earliness and yield of brinjal hybrids. Journal Soils Crops, v.4, p.217-222, 2003.

Silva, E. M.; Lima, C. J. G. S.; Duarte, S. N.; Barbosa, F. S.; Maschio, R. Níveis de salinidade e manejo da fertirrigação sobre características da berinjela cultivada em ambiente protegido. Revista Ciência Agronômica, v.44, p.150-158, 2013.

Trani, P. E.; Tiveli, S. W.; Carrijo, O. A. Fertirrigação em hortaliças. 2.ed. rev. atual. Campinas: Instituto Agronômico, 2011.51p. Série Tecnologia APTA. Boletim Técnico IAC, 196

Unlukara, A.; Kurunç, A.; Kesmez, G. D.; Yurtseven, E.; Suarez, D. Effects of salinity on eggplant (Solanum Melongena L.) growth and evapotranspiration. Journal of Irrigation and Drainage, v.59, p.203-214, 2010.

Wu, X. X; Ding, H. D.; Zhu, Z. W.; Yang, S. J.; Zha, D. S. Effects of 24-epibrassinolide on photosynthesis of eggplant (Solanum melongena $\mathrm{L}$.) seedlings under salt stress. African Journal of Biotechnology, v.11, p.8665-8671, 2012. 